\title{
ON EQUITABLE COLORING OF BOOK GRAPH FAMILIES
}

\author{
M. BARANI ${ }^{1}$, M.VENKATACHALAM ${ }^{2}$, and K. RAJALAKSHMI ${ }^{3}$ \\ ${ }^{1,2} \mathrm{PG}$ and Research Department of Mathematics, Kongunadu Arts and Science College, \\ Coimbatore-641029 INDIA \\ ${ }^{3}$ Department of Science and Humanities, Sri Krishna College of Engineering and Technology, \\ Coimbatore-641008, INDIA
}

\begin{abstract}
A proper vertex coloring of a graph is equitable if the sizes of color classes differ by atmost one. The notion of equitable coloring was introduced by Meyer in 1973. A proper $h$-colorable graph $K$ is said to be equitably h-colorable if the vertex sets of $K$ can be partioned into $h$ independent color classes $V_{1}, V_{2}, \ldots, V_{h}$ such that the condition ||$V_{i}|-| V_{j}|| \leq 1$ holds for all different pairs of $i$ and $j$ and the least integer $h$ is known as equitable chromatic number of $K$. In this paper, we find the equitable coloring of book graph, middle, line and central graphs of book graph.
\end{abstract}

\section{INTRODUCTION}

The idea of equitable coloring was discovered by Meyer [4] in 1973. Hajmal and Szemeredi [3] proved that graph $K$ with degree $\Delta$ is equitable h-colorable, if $h \geq \Delta+1$. Later Equitable Coloring Conjecture for bipartite graphs was proved. Equitable vertex coloring of corona graphs is NP- hard.

The graphs considered here are simple. Vertex coloring is a particular case of Graph coloring. The collection of vertices receiving same color is known as color class. A proper $h$-colorable graph $K$ is said to be equitably $h$-colorable if the vertex sets of $K$ can be partitioned into $h$ independent color classes $V_{1}, V_{2}, \ldots, V_{h}$ such that the condition ||$V_{i}|-| V_{j}|| \leq 1$ holds for all different pairs of $i$ and $j$ [1]. And the least integer $h$ is known as equitable chromatic number of $K$ [1]. Here we found equitable coloring of book graph, middle, line and central graphs of book graph.

2020 Mathematics Subject Classification. 05C15, 05C76.

Keywords and phrases. Equitable coloring, book graph, middle graph, line graph, central graph Submitted via ICCSPAM 2020.

凹baranibe2013@gmail.com; venkatmaths@gmail.com-Corresponding author; rajalakshmikandhasamy@gmail.com

(D) 0000-0002-4373-5117; 0000-0001-5051-4104; 0000-0003-4737-2656. 


\section{Preliminaries}

Line graph [2] of $K, L(K)$ is attained by considering the edges of $K$ as the vertices of $L(K)$. The adjacency of any two vertices of $L(K)$ is a consequence of the corresponding adjacency of edges in $K$.

Middle graph [5] of $K, M(K)$ is attained by adding new vertex to all the edges of $K$. The adjacency of any two new vertices of $M(K)$ is a consequence of the corresponding adjacency of edges in $K$ or adjacency of a vertex and an edge incident with it.

Central graph [6] of $K, C(K)$ is attained by the insertion of new vertex to all the edges of $K$ and connecting any two new vertices of $K$ which were previously non-adjacent.

The q-book graph is defined as the graph Cartesian product $S_{(q+1)} \times P_{2}$, where $S_{q}$ is a star graph and $P_{2}$ is the path graph.

\section{Results}

\subsection{On Equitable Coloring of Middle Graph of Book Graph.}

- Order of $M\left(B_{q}\right)$ is $5 q+3$

- Number of incidents of $M\left(B_{q}\right)$ is $q^{2}+9 q+2$

- Maximum degree of $M\left(B_{q}\right)$ is $2(q+1)$

- Minimum degree of $M\left(B_{q}\right)$ is 2

\section{Algorithm A}

Input: The value ' $q$ ' of $B_{q}$, for $q \geq 3$

Outcome: Equitably colored $V\left[M\left(B_{q}\right)\right]$

Procedure:

$$
\begin{aligned}
& \text { start } \\
& \{ \\
& V_{a}=\{g, h, z\} ; \\
& C(g)=C(h)=1 ; \\
& C(z)=q+2 ; \\
& \text { for } s=1 \text { to } q \\
& \{ \\
& V_{b}=\left\{g_{s}, h_{s}\right\} \\
& C\left(g_{s}\right)=s ; \\
& C\left(h_{s}\right)=s ; \\
& \} \\
& \text { for } s=1 \text { to } q \\
& \{ \\
& V_{c}=\left\{k_{s}, l_{s}\right\} \\
& C\left(k_{s}\right)=s+1 ; \\
& C\left(l_{s}\right)=s+1 ; \\
& \}
\end{aligned}
$$




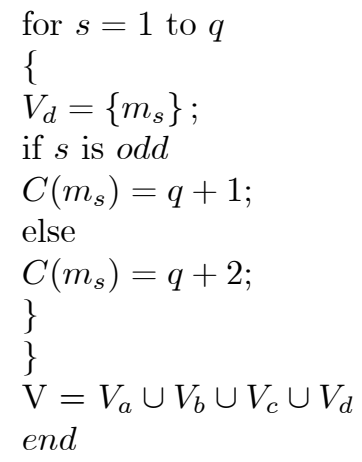

Theorem 3.1. For any book graph $M\left(B_{q}\right)$ the equitable chromatic number,

$$
\boldsymbol{\chi}_{=}\left[\mathbf{M}\left(\mathbf{B}_{\mathbf{q}}\right)\right]=\mathbf{q}+\mathbf{2}, \forall q \geq 3
$$

Proof. For $q \geq 3, V\left(B_{q}\right)=\left\{g, h, g_{s}, h_{s}: 1 \leq s \leq q\right\}$.

$V\left[M\left(B_{q}\right)\right]=\{g, h, z\} \cup\left\{g_{s}: 1 \leq s \leq q\right\} \cup\left\{h_{s}: 1 \leq s \leq q\right\} \cup\left\{k_{s}: 1 \leq s \leq q\right\} \cup\left\{l_{s}:\right.$ $1 \leq s \leq q\} \cup\left\{m_{s}: 1 \leq s \leq q\right\}$, where $z, k_{s}, l_{s}$ and $m_{s}$ are the subdivision of the edges $g h, g g_{s}, h h_{s}$ and $g_{s} h_{s}$ respectively.

Let us consider $V\left[M\left(B_{q}\right)\right]$ and the color set $\mathrm{C}=\left\{c_{1}, c_{2}, \ldots, c_{q+2}\right\}$. Assign the equitable coloring by Algorithm A. Therefore,

$$
\chi_{=}\left[M\left(B_{q}\right)\right] \leq q+2 .
$$

And since, there exists a maximal induced complete subgraph of order $q+2$ by the vertices $z, g, k_{s}$ and therefore $\chi_{=}\left[M\left(B_{q}\right)\right] \geq q+2$.

$c_{1}, c_{2}, \ldots, c_{q+2}$ are independent sets of $M\left(B_{q}\right)$. And ||$c_{i}|-| c_{j}|| \leq 1$, for every different pair of $i$ and $j$. Hence,

$$
\chi_{=}\left[\mathbf{M}\left(\mathbf{B}_{\mathbf{q}}\right)\right]=\mathbf{q}+\mathbf{2} .
$$

\subsection{On Equitable Coloring of Central Graph of Book Graph. Features of Central Graph of Book Graph}

- Order of $C\left(B_{q}\right)$ is $5 q+3$

- Number of incidents of $C\left(B_{q}\right)$ is $2\left(q^{2}+3 q+1\right)$

- Maximum degree of $C\left(B_{q}\right)$ is $2 q+1$

- Minimum degree of $C\left(B_{q}\right)$ is 2

Algorithm B

Input: The value ' $q$ ' of $B_{q}$, for $q \geq 3$

Outcome: Equitably colored $V\left[C\left(B_{q}\right)\right]$

Procedure:

start 


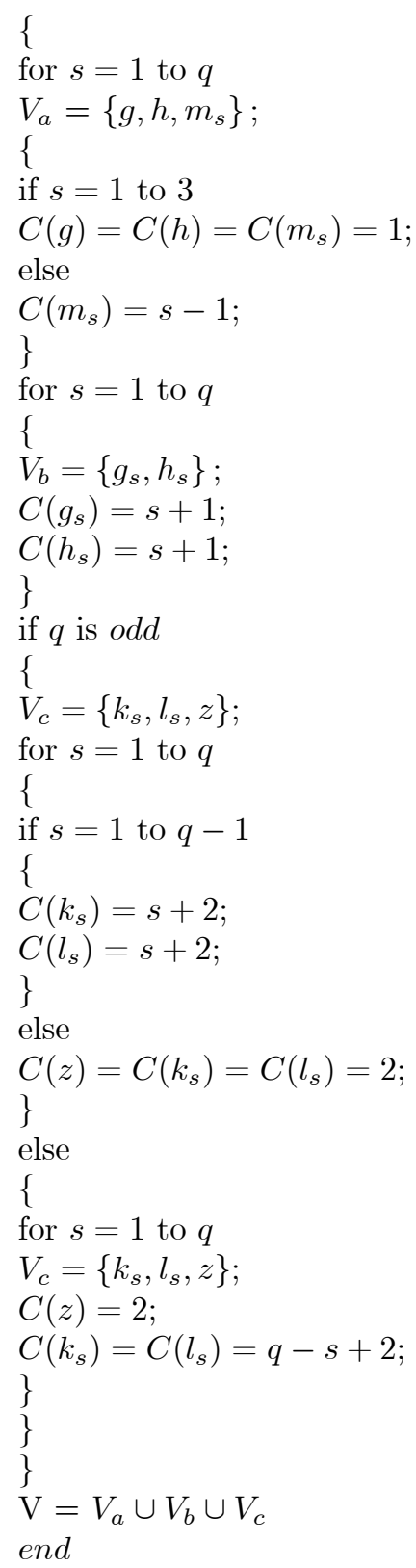

Theorem 3.2. For any book graph $C\left(B_{q}\right)$ the equitable chromatic number,

$$
\chi_{=}\left[\mathbf{C}\left(\mathbf{B}_{\mathbf{q}}\right)\right]=\mathbf{q}+\mathbf{1}, \forall \mathbf{q} \geq \mathbf{3}
$$


Proof. For $q \geq 3$,

$$
\begin{aligned}
& V\left(B_{q}\right)=\left\{g, h, g_{s}, h_{s}: 1 \leq s \leq q\right\} . \\
V\left[C\left(B_{q}\right)\right]= & \{g, h, z\} \cup\left\{g_{s}: 1 \leq s \leq q\right\} \cup\left\{h_{s}: 1 \leq s \leq q\right\} \\
\cup\left\{k_{s}:\right. & 1 \leq s \leq q\} \cup\left\{l_{s}: 1 \leq s \leq q\right\} \cup\left\{m_{s}: 1 \leq s \leq q\right\},
\end{aligned}
$$

where $z, k_{s}, l_{s}$ and $m_{s}$ are the subdivision of the edges $g h, g g_{s}, h h_{s}$ and $g_{s} h_{s}$ respectively.

Let us consider $V\left[C\left(B_{q}\right)\right]$ and the color set $\mathrm{C}=\left\{c_{1}, c_{2}, \ldots, c_{q+1}\right\}$. Assign the equitable coloring by Algorithm B. Therefore,

$$
\chi_{=}\left[C\left(B_{q}\right)\right] \leq q+1
$$

And $\chi\left[C\left(B_{q}\right)\right]=q+1$. That is, $\chi_{=}\left[C\left(B_{q}\right)\right] \geq \chi\left[C\left(B_{q}\right)\right]=q+1$. Therefore,

$$
\chi_{=}\left[C\left(B_{q}\right)\right] \geq q+1 \text {. }
$$

$c_{1}, c_{2}, \ldots, c_{q+1}$ are independent sets of $C\left(B_{q}\right)$. And ||$c_{i}|-| c_{j}|| \leq 1$, for every different pair of $i$ and $j$. Thus,

$$
\chi_{=}\left[C\left(B_{q}\right)\right]=q+1 \text {. }
$$

\subsection{On Equitable Coloring of Line Graph of Book Graph.}

- Order of $L\left(B_{q}\right)$ is $3 q+1$

- Number of incidents of $L\left(B_{q}\right)$ is $q(q+3)$

- Maximum degree of $L\left(B_{q}\right)$ is $2 q$

- Minimum degree of $L\left(B_{q}\right)$ is 2

Algorithm C

Input: The value ' $q$ ' of $B_{q}$, for $q \geq 3$

Outcome: Equitably coloring $V\left[L\left(B_{q}\right)\right]$

Procedure:

begin

\{

for $s=1$ to $q$

\{

$V_{a}=\{g, z\} \cup\left\{m_{s}\right\}$;

$C\left(m_{s}\right)=s$

$C(z)=C(g)=1$

\}

for $s=1$ to $q$

\{

$V_{b}=\left\{k_{s}, l_{s}\right\}$

$C\left(k_{s}\right)=s+1$

$C\left(l_{s}\right)=s+1$

\} 
$\mathrm{V}=V_{a} \cup V_{b}$

end

Theorem 3.3. For any book graph $L\left(B_{q}\right)$ the equitable chromatic number,

$$
\chi_{=}\left[\mathbf{L}\left(\mathbf{B}_{\mathbf{q}}\right)\right]=\mathbf{q}+\mathbf{1}, \forall \mathbf{q} \geq \mathbf{3}
$$

Proof. For $q \geq 3$,

$$
V\left(B_{q}\right)=\left\{g, h, g_{s}, h_{s}: 1 \leq s \leq q\right\} .
$$

The edge set of $B_{q}$ is $\left\{z, k_{s}, l_{s}, m_{s}: 1 \leq s \leq q\right\}$ where $z$ be the edge corresponding to the vertices $g h$, each $k_{s}$ be the edge corresponding to the vertex $g g_{s}$, each edge $l_{s}$ be the edge corresponding to the vertex $h h_{s}$, each edge $m_{s}$ be the edge corresponding to the vertex $g_{s} h_{s}$. By the definition of line graph, the edge set of line graph is converted into vertices of $L\left(B_{q}\right)$.

$$
\begin{aligned}
& V\left[L\left(B_{q}\right)\right]=\{z\} \cup\left\{k_{s}: 1 \leq s \leq q\right\} \cup\left\{l_{s}: 1 \leq s \leq q\right\} \\
& \cup\left\{m_{s}: 1 \leq s \leq q\right\} .
\end{aligned}
$$

Let us consider the $V\left[L\left(B_{q}\right)\right]$ and the color set $C=\left\{c_{1}, c_{2}, \ldots, c_{q+1}\right\}$. Assign the equitable coloring by Algorithm C. Therefore,

$$
\chi_{=}\left[L\left(B_{q}\right)\right] \leq q+1
$$

And since, there exists a maximal induced complete subgraph of order $q+1$ by the vertices $z, k_{s}$ and therefore

$$
\chi_{=}\left[L\left(B_{q}\right)\right] \geq q+1 .
$$

$c_{1}, c_{2}, \ldots, c_{q+1}$ are independent sets of $L\left(B_{q}\right)$. And ||$c_{i}|-| c_{j}|| \leq 1$, for every different pair of $i$ and $j$. Thus,

$$
\chi_{=}\left[L\left(B_{q}\right)\right]=q+1 .
$$

\subsection{On Equitable Coloring of Book Graph. Features of Book Graph.}

- Order of $B_{q}$ is $2(q+1)$

- Number of incidents of $B_{q}$ is $3 q+1$

- Maximum degree of $B_{q}$ is $q+1$

- Minimum degree of $B_{q}$ is 2

\section{Algorithm D}

Input: The value 'q' of $B_{q}$, for $\mathrm{q} \geq 3$

Outcome: Equitably colored $V\left(B_{q}\right)$

Procedure:

$$
\begin{aligned}
& \text { start } \\
& \text { for } s=1 \text { to } q \\
& \{ \\
& V_{a}=\left\{g_{s}, h\right\} ; \\
& \mathrm{C}(\mathrm{h})=1 ;
\end{aligned}
$$




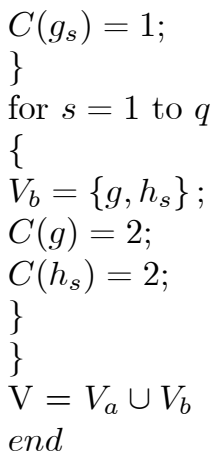

Theorem 3.4. For any book graph $B_{q}$ the equitable chromatic number,

$$
\chi_{=}\left(\mathbf{B}_{\mathbf{q}}\right)=\mathbf{2}, \forall q \geq 3 \text {. }
$$

Proof. For $n \geq 3$,

$$
V\left(B_{q}\right)=\{g, h\} \cup\left\{g_{s}: 1 \leq s \leq q\right\} \cup\left\{h_{s}: 1 \leq s \leq q\right\} .
$$

Let us consider the $V\left(B_{q}\right)$ and the color set $\mathrm{C}=\left\{c_{1}, c_{2}\right\}$. Assign the equitable coloring by Algorithm D. Therefore,

$$
\chi_{=}\left(B_{q}\right) \leq 2 .
$$

And since, there exists a maximal induced complete subgraph of order 2 in $B_{q}$ (say path $P_{2}$ ). Therefore,

$$
\chi_{=}\left(B_{q}\right) \geq 2
$$

$c_{1}, c_{2}$ are independent sets of $B_{q}$. And ||$c_{i}|-| c_{j}|| \leq 1$, for every different pair of $i$ and $j$. Hence,

$$
\chi_{=}\left(B_{\mathbf{q}}\right)=2 .
$$

\section{REFERENCES}

[1] Furmanczyk, H., Equitable coloring of Graph products, Opuscula Mathematica, Vol 26. No.1, (2006).

[2] Harary, F., Graph theory, Narosa Publishing home, New Delhi, 1969.

[3] Hajnal, A., Szemeredi, E., Proof of a conjecture of Endos, in: Combinatorial theory and its applications, Colloq. Math. Soc. Janos Bolyai, 4 (2) (1970), 601-623.

[4] Meyer, W., Equitable coloring, Amer. Math. Monthly, 80, (1973).

[5] Michalak, D., On middle and total graphs with coarseness number equal 1, Springer Verlag Graph Theory, Lagow Proceedings, Berlin Heidelberg, New York, Tokyo, (1981), 139-150.

[6] Vernold Vivin, J., Harmonious coloring of total graphs, n-leaf, central graphs and circumdetic graphs, Bharathiar University, Ph.D Thesis, Coimbatore, India, 2007. 